\title{
Model reduction in large chemical systems- an alternative method applying discrete approximation
}

\author{
by \\ Magne Fjeld @ †
}

\section{Introduction}

In some areas of science such as in the studies of large dynamical systems, there has been considerable interest over more than 50 years in simplifying system description by reducing the order of the set of differential equations. The purpose has been either to make an order reduction, a simplification of the system, or to make an asymptotical order reduction valid in some neighbourhood of the steady state, as the system response approaches the steady state.

In a bottom-up approach of systems modelling, a system is modeled using first-principle physical and chemical laws, taking into account as much detailed knowledge as available. However, this approach may lead to large-scale models with huge numbers of variables. For efficient simulations these high-dimensional models are inappropriate, in particular if they involve multiple time scales (with stiff numerical solution properties) such that $150 \mathrm{hrs}$ of supercomputer time reportedly were required to make calculations of a steady axisymmetric, methane-air diffusion flame. Combustion processes may have 600 species and 2500 reaction equations (Chevalier et.al 1992).

For such high-dimensional models model reduction are welcomed into application. A central issue of model reduction is to address the discrepancy between the need to develop detailed high-dimensional multi-timescale models (e.g. in chemical kinetics) and the inefficiency of their use in computationally demanding numerical simulations. The ultimate goal of most model reduction techniques, however, is to find a lower dimensional approximation which contains all the essential information to still describe the system accurately enough for the purpose of the uses of model.

Considering models of chemical kinetics, Gorban and Karlin suggested that model reduction techniques could be classified according to the following purposes (Gorban, Karlin, Springer 2005):

$\dagger$ Reprint only by written consent by the author. E-mail: magnefjeld@gmail.com 
1.Reduce the number of species. This, in turn, can be achieved in two ways:

- eliminate inessential species, or

- lump some of the species into integrated components

2. Reduce the number of reactions.

3. Decompose the motions into fast and slow motions, into independent (almostindependent) and slaved motions, etc.

However, in simulations of chemical processes often all species are relevant for the properties of interest and therefore may have to be considered, not only the ones included in the reduced mechanism. Hence the concentrations of the species of the full mechanism need to be automatically calculated as functions of the species of the reduced mechanism. This is an automatic species reconstruction, and is implemented in most of the model reduction algorithms, independently of the concepts the methods are based on.

Theory of invariance plays a role in reduction of model complexity. Invariance theory in mathematics is at least 170 yrs old, introduced by Cayley (1845), in "On the Theory of Linear Transformations " Classically, the term "invariant theory" refers to the study of invariant algebraic forms for the action of linear transformations.

Now to the model reduction methods in chemical engineering.

For closed and lumped systems in chemical engineering, the concept of chemical invariants were introduced by Aris (1965), Amundson (1966), and Froment (1967), Aris (1968) derived equations for chemical invariants. Aris (1968) in his mathematically tilted "Prolegomena" paper, stated for a reaction system of $S$ species and R independent reactions ( according to his Theorem 9, "..the composition may be expressed in terms of extents and invariants..", and Theorem 8 , "...there are S-R independent concentrations....that are invariant under reaction".

The notions of chemical invariants and variants can be applied to model reduction, perhaps most advantageously with few reactions but complex chemistry, and where the equipment part (defining flows) is usually either defined by a well stirred batch reactor, or an ideal plug flow pipe reactor. In the case of open systems, invariants may be considered by application of the modified concept "asymptotic invariants", which was first suggested by Fjeld ( contrary to most references, in 1968), see Fjeld (1968), submitted to the journal in 1967, see also Fjeld (1969), Fjeld(1971). 
Following these ideas, analytic procedures were developed to study the mathematical structure of the reactor, for the purpose of singling out the reaction variant and the reaction invariant parts of the system. One purpose of such an approach has been simplification in simulation and control of systems of medium complexity (which may have a high number of state variables due to complex kinetics, but with relatively few reaction equations).

The ideas were elaborated much further, both published in Ph.D thesises, and in journal papers, such as by Asbjørnsen \& Fjeld (1970). Some new ideas on application of projection operators, the analysis of observabilty and controllability as related to invariants, were developed by Fjeld et al (1974).

\section{Asymptotic invariants and system decomposition to variants and invariants. Preliminaries.}

It was suggested by Fjeld (1968) that in open systems there exists asymptotic invariants, a linear combination of the number of moles $\mathbf{m}$ of each chemical species, such that

$\mathrm{d} \mathbf{m}(\mathrm{t}) / \mathrm{dt}=-\lambda \mathbf{m}+\mathbf{C} \quad, \mathbf{C}=$ a constant vector

Why Invariants ? - this question has been answered by the Ydstie Research Group, see Aggarwal (2007). Some of the advantages using invariants are that they

a Provide a minimal state representation

a Allow the use of the structure of physical processes for modeling.

a Separate the model into modules of thermodynamics and transport phenomenons.

a Provide a basis for stability analysis

a Provide model reduction

A communication paper by Fjeld (1969) in Chem.Eng. Science suggested a tri-diagonal for the thermodynamic set ot equations, based on a transformation of the concentrations of species and the temperature in a CSTR.

Fjeld $(1968,1969,1971)$ used the stoichiometric matrix $\mathrm{N}$, through a decomposition method, supported by the tridiagonal form, to construct a linear transformation of the concentration states $\mathbf{m}(\mathrm{t})$ to the reaction-variant states $\mathbf{y}(\mathrm{t})$, and the reaction-invariant states $\mathbf{z}(\mathrm{t})$. This standard approach breaks down if axial diffusion is present in the reactor, and this fact will be shown in the following sections. Next, an an alternative modelling route is presented, to account for axial diffusion, and still maintain the simple decomposition of the stoichiometric matrix as a method to arrive at reaction-invariant states. 


\section{The tridiagonal form}

The starting point to develop the transformations to find invariants, is the tridiagonal form of the dynamic reaction equations, which is summarized below. The autonomous part of the linearized CSTR system around the steady state is of the following form, where $\mathbf{N}$ is the stoichiometric matrix, and $\mathbf{r}$ is the reaction rate vector,

$\mathrm{d} \Delta \mathbf{m} / \mathrm{dt}=-(\mathrm{q} / \mathrm{V}) \Delta \mathbf{m}+\mathbf{N}^{\top}(\delta \mathrm{r}(\mathbf{m}) / \delta \mathrm{m}) \Delta \mathbf{m}$

where the system matrix is $\quad G=-(q / V) \mathbf{I}+\mathbf{N}^{\top}(\delta \mathbf{r}(\mathbf{m}) / \delta \mathbf{m})$

If $\mathrm{N}$ is of full rank (the $\mathrm{R}$ reactions are independent), then Fjeld (1971) found that $\mathrm{G}$ can be transformed, under suitable change of basis, to the tridiagonal form

$$
\left[\begin{array}{ll}
Y & Z \\
0 & F
\end{array}\right]
$$

or, the following associated decomposed state space description,

$$
\left(\begin{array}{c}
\dot{y} \\
\dot{z}
\end{array}\right)=\left[\begin{array}{cc}
Y(v) & Z(v) \\
0 & F
\end{array}\right]\left(\begin{array}{l}
y \\
z
\end{array}\right)
$$

where $\mathbf{y , z}$ are vectors, the vector $\mathbf{v}$ is a function of $\mathbf{m}$, and $\mathrm{F}, \mathrm{Y}$, and $\mathrm{Z}$ matrices of matching dimensions.

As pointed out by Fjeld (1969) the transformation to eq (2) from the state variables consists of linear combinations of the components of $\mathbf{v}$, where the decomposed $\mathbf{v}$ into $\mathbf{y}$ and $\mathbf{z}$ constitute the reaction variant and reaction invariant dynamics respectively. Further, for a CSTR, $\mathbf{F}=\mathbf{I} / \mathrm{T}, \mathrm{T}$ is the mean residence time, $\mathbf{I}$ is the unity diagonal matrix, $\operatorname{dim}(\mathbf{y})=R$ (the dimension of the reaction variant vector), $\operatorname{dim}(\mathbf{z})=\mathrm{S}-\mathrm{R}$ (the dimension of the reaction invariants). In a closed system $\mathbf{F}$ will be a matrix with zero entries. In constant temperature cases, the heat balance equation is not present. Then the number of invariants is $S-R, S$ is the number of chemical species, and $R$ is the number of linearly independent reactions, equal to the number of variants. 
The decoupled vector $\mathbf{z}$ (either an asymptotic or true invariant) forms a "disturbance" to the reaction variant part of the system, however, with $\lim (\mathbf{z})=\mathbf{0}$ as $t->\infty$ in the autonomous set eq(2), starting wth $\mathbf{z}(\mathbf{0})$, and decaying exponentially in a CSTR (unless it is a flow variant).

Hence we observe that $\mathbf{z}$ is invariant under reaction, and Fjeld (1969) proved that $G$ has a stable eigensubspace of dimension $S-R$, which is the orthogonal complement $\mathbf{R}_{\mathbf{R}}$ of $\mathbf{R}_{\mathbf{R}}$. For symbols and indices, see section 2 below that follows ( $S$ is the number of species, $R$ is the number of independent reactions). Although the dimension of the two subspaces (the reaction variant and the reaction invariant subspaces) are fixed, many different choices of coordinates are possible. Or differently stated, there are many transformations available that lead to the two subspaces.

As a consequence, this state transformation allows a reduction of the dimension of the state space when it suffices to study the reaction variant subspace only. One purpose may be analysis of local stability of the reaction system. Or the reduction may simplify simulations and other areas of the engineering cybernetics sphere of theory and applications. It was shown (Fjeld 1969) for the first time that both the asymptotic and true invariants could be unconditionally deleted from the system description, in the case of analysis of asymptotic stability ("stability in the small”).

Asbjørnsen (1972) concludes in his study on invariants in CSTRs and tubular reactors that "the analysis and simulation of the dynamics of continuous chemical reactors for multicomponents mixtures are greatly simplified by the use of asymptotic invariants".

\section{Some definitions:}

Reaction \& Flow Variants:

Variables that vary with time due to the effects of chemical reactions and physical flows

Flow Variants \& Reaction Invariants:

Variables that vary with time due to other rate processes (mass transfer, inlets/outlet), but are independent of chemical reactions

Reaction \& Flow Invariants:

Variables that do not vary with time and stay constant during the course of the reaction 


\section{Reaction network and stoichiometry}

Consider the linearly independent stoichiometric chemical reaction equations

$$
\underline{\mathbf{N}} \underline{\mathbf{X}}=0 ; \quad \underline{\mathbf{N}}=\left(\mathrm{n}_{\mathrm{ij}}\right) ; \mathbf{X}^{\top}=\left(\mathrm{x}_{1}, \mathrm{x}_{2}, \ldots \ldots . . \mathrm{x}_{\mathrm{S}}\right)
$$

where $i=1,2, \ldots . R ; j=1,2, \ldots . . S ; x_{j}$ denotes the $j^{\text {th }}$ reacting species, $S$ is the total number of reacting species.

Definitions:

a reactant is a species that appears on the left-hand side of at least one reaction.

aA product is a species that appears on the right-hand side of at least one reaction.

ay convention, reactants and products have negative and positive entries respectively, in the stoichiometric reaction equations.

The R X S stoichiometric matrix $\mathbf{N}$ becomes

$$
\begin{aligned}
& \mathrm{n}_{11} \quad \mathrm{n}_{12} \quad \ldots \ldots \ldots \ldots . \quad \mathrm{n}_{1 \mathrm{~S}} \\
& \begin{array}{lllll}
\mathrm{n}_{21} & \mathrm{n}_{22} & \ldots \ldots \ldots \ldots \ldots . & \mathrm{n}_{2 \mathrm{~S}}
\end{array} \\
& \mathbf{N}=
\end{aligned}
$$

$\mathrm{n}_{\mathrm{R} 1} \quad \mathrm{n}_{\mathrm{R} 2} \quad \cdots \cdots \cdots \cdots \cdots \mathrm{n}_{\mathrm{RS}}$

The number of species is $\mathrm{S}$, the number of fully independent reactions is assumed to be $R$.

\section{Reaction kinetics}

Let the molar concentration $c_{j}(k)$ be defined as

$$
\begin{aligned}
& m_{j}(k) \quad=c_{j}(k) \quad \text { for } j=1,2,3 \ldots . . S \\
& \mathrm{~V}(\mathrm{k})
\end{aligned}
$$

where $m_{j}$ is the number of moles of the $j^{\text {th }}$ species, ad $V$ is the reactor volume. The unit is usually given in $\left(\right.$ mole $\left.^{-1}\right)$, or abbreviated $(M)$.

The reaction rates are given by

$$
\begin{aligned}
& r_{i}\left(\underline{c}, \theta_{i}\right)=x_{i} \quad r_{i j}\left(c_{j}, \quad \theta_{i, j}\right) \\
& \theta_{i}=\operatorname{col}\left(\mathcal{X}_{i}, \quad \theta_{i 1}, \theta_{i 2} \ldots \ldots, \theta_{i S}\right) \quad ; \mathbf{r}=\mathbf{r}\left(\begin{array}{cc}
\mathbf{c}, & \boldsymbol{\theta}
\end{array}\right)
\end{aligned}
$$


where $\theta_{i, j}$ are temperatures.

Rank (N) = R follows from the independency of the equations (2); If not $\mathbf{N}$ is of full rank, a decomposition (with ordering of equations) can in many cases be made such that this is indeed the case for the remaining full-ranked $R$ equations defined by eq(2).

The invariance property is due to the structure of the stoichiometric matrix. Embedded in this matrix, is satisfaction of conservation of atoms. Indeed, let $\boldsymbol{\phi}$ be an $S \times A$ matrix, where $\phi_{i j}$ indicates the number of atoms of the ith type up to $A$, in the jth chemical species $j$ up to $S$. $A$ is the number of quantities conserved. Any row of $\mathbf{N}$ must satisfy the conservation equation for conservation of the $A$ conserved quantities, which can be compactly expressed for all rows of $\mathrm{N}$, i..e all of the $\mathrm{R}$ reactions,

$$
\mathbf{N} \boldsymbol{\phi}=\mathbf{0}_{\mathrm{RXA}} \text { or } \boldsymbol{\phi}^{\top} \mathbf{N}^{\top}=\mathbf{0}_{\mathrm{A} \times \mathrm{R}}
$$

We conclude that the conservation law is equivalent to eq(6), i.e. the reaction stoichiometrics must lie in the null space of the reaction-invariance matrix $\boldsymbol{\phi}^{\top}$.

\section{The dynamic model for a CSTR with inlet and outlet streams}

\subsection{Mass balance}

With a homogeneous stirred tank reaction system, with $p$ inlet streams, and one outlet stream, $\mathrm{S}$ species, and $\mathrm{R}$ independent reactions, the mole balance is

$$
\mathrm{d}(\mathrm{V} \mathbf{c}) / \mathrm{dt}=\mathbf{N}^{\top} \mathbf{r}(\mathbf{c}, \mathrm{T})+\mathbf{C}_{\text {in }} \mathbf{q}_{\text {in }}-\mathrm{q}_{\text {out }} \mathbf{c} \quad(\mathrm{V} \mathbf{c})(\mathrm{t}=0)=\mathrm{V}_{0} \mathbf{c}_{0}
$$

where the inlet Sxp molar concentrations, $C_{\text {in }}=\left(C^{1}{ }_{\text {in }}, C^{2}\right.$ in, .$\left.c^{p}{ }_{\text {in }}\right)$

$\mathbf{r}$ is a function of both $\mathbf{c}$ and $T$, and assumed to be an Arrhenius function of $T$.

The equation for $\mathrm{dV} / \mathrm{dt}$ needs to be established through the continuity equation, a simple exercise which is left out here.

\subsection{The heat balance equation}

$\mathrm{d}\left(\mathrm{mc}_{\mathrm{p}} \mathrm{T}\right) / \mathrm{dt}=\mathrm{V}\left(-\boldsymbol{\Delta} \mathbf{h}_{\mathrm{R}}{ }^{\top}\right) \mathbf{r}(\mathbf{c}, \mathrm{T})+\mathbf{T}_{\text {in }}{ }^{\top} \mathbf{C}_{\mathrm{p}, \text { in }} \mathrm{Y}_{\text {in }}-\mathrm{T} \mathrm{c}_{\mathrm{p}} \mathrm{Y}_{\text {out }}+\mathrm{d} \mathrm{Q}_{\mathrm{ext}} / \mathrm{dt}$

where $c_{p}$ is the specific heat capacity, $\Delta h_{R}$ the R-dimensional vector of reaction enthalpies, $\mathbf{T}_{\text {in }}$ is the $p$-dimensional inlet temperature vector, $\mathbf{C}_{p, \text { in }}$ is the p-dimensional diagonal matrix with elements being specific heat capacities of the inlet streams with flows $\gamma_{\text {in }}$. There is only one outlet stream with flow $\gamma_{\text {out }} \mathrm{dQ}_{\text {exx }} / \mathrm{dt}$ is the external heat power, and $\mathrm{T}_{0}$ the initial temperature. 
4. Reaction and flow variants invariants in dynamic models

4.1 Finding the invariant space through simple partitioning of the stoichiometric matrix, applying extents of reaction as coordinates

A simple partitioning method is

$\mathrm{dc} / \mathrm{dt}=\mathbf{M}^{\boldsymbol{\top}} \mathbf{r}(\mathbf{c})+1 / \mathrm{T}\left(\mathbf{c}_{\mathrm{in}}-\mathbf{c}\right) \quad\left(\mathbf{M}^{\boldsymbol{\top}}=\mathbf{N}^{\boldsymbol{\top}} / \mathrm{V}\right)$

and for a batch reaction

$\mathrm{d} \mathbf{c} / \mathrm{dt} \quad \mathbf{M}^{\top} \mathbf{r}(\mathbf{c})$

and the column vectors $\mathbf{m}_{\mathbf{i}}(\mathrm{i}=1, \ldots \ldots . \mathrm{S}) ; \operatorname{dim} \mathbf{M}^{\boldsymbol{\top}}=\mathrm{S} \times \mathrm{R}$

$M=\left[m_{1} m_{2} m_{3} \ldots \ldots \ldots \ldots \ldots m_{R} \quad m_{R+1} \ldots \ldots \ldots \ldots m_{S}\right]=\left[\begin{array}{l}M_{1} M_{2}\end{array}\right]$

$\operatorname{dim} \mathbf{M}=\mathrm{R} \times \mathbf{S} ; \operatorname{dim} \mathbf{M}_{\mathbf{1}}=\mathrm{RxR} ; \operatorname{dim} \mathbf{M}_{\mathbf{2}}=\mathrm{Rx}(\mathrm{S}-\mathrm{R})$

For a batch system, defining $\mathbf{c}(0)=\mathbf{c}_{0} ; \mathbf{c}_{1}(\mathbf{0})=\mathbf{c}_{10}, \mathbf{c}_{2}(\mathbf{0})=\mathbf{c}_{20}$

Then using the extent of reaction vector $\xi, \operatorname{dim}(\xi)=R$

$\mathbf{d c}_{\mathrm{j}}=\mathbf{m}_{\mathrm{j}}^{\top} \mathrm{d} \boldsymbol{\xi} \quad$ since $\boldsymbol{\xi}(0)=\mathbf{0}$

Hence,

$$
\begin{array}{ll}
c_{1}(t)-c_{1}(0)=c_{1}(t)-c_{10}= & M_{1}^{\top} \xi \\
c_{1}-c_{10}=M_{1}^{\top} \xi & \operatorname{dim}\left(c_{1}\right)=R \\
c_{2}-c_{20}=M_{2}^{\top} \xi & \operatorname{dim}\left(c_{2}\right)=S-R
\end{array}
$$

Since $\mathbf{M}_{\mathbf{1}}$ is nonsingular, the extent of reaction $\boldsymbol{\xi}(\mathrm{t})$ is uniquely determined by $\mathbf{c}_{\mathbf{1}}$

$$
\mathrm{c}(\mathrm{t})-\mathrm{c}_{0}=\mathrm{M}^{\mathrm{T}} \boldsymbol{\xi}(\mathrm{t})=\mathbf{M}^{\mathrm{T}} \int_{0}^{t} r(c(t)) d t
$$

for any $\mathbf{c}_{1}-\mathbf{c}_{10} \geq \mathbf{0}$ (note, as chemical species, $\mathbf{c}(\mathrm{t})$ is a vector with nonzero entries $\mathbf{c}_{\mathbf{i}}$ $\geq 0$ ). 
For an open reactor as a CSTR, see section 4.2, where the simple approach above is extended.

It should be noted, that based on chemistry insights, it may be desirable to apply a different variant vector than one based on any partioning and reordering of the stoichiometric matrix. Indeed, applying the extent of reaction vector, is one such particular choice which may be meaningful for the process analyst, given by

$$
\xi(t)=\left(M^{T}\right)^{-1}\left(c(t)-c_{0}\right)
$$

Any invertible transformation operating on $\boldsymbol{c}_{1}$ may be applied to transform $\boldsymbol{c}_{1}$ into a variant that suites the purpose of the modelling better (control, measurements, estimation). Indeed, Waller and Makila (1981) suggested that the variants should be chosen on other grounds than orthogonality, by some transformation $\mathbf{L}$ of the state variables, not purely algebraic, but based on chemical insights in the reaction taking place,

$$
\mathbf{v}=\mathbf{L C}
$$

Waller and Makila state that the most obvious $\mathbf{L}$ is simply $\mathbf{L}=\mathbf{M}^{\mathbf{\top}}{ }_{\mathrm{RxS}}$.

When, as advised by Fjeld (1971), see section 4.2 in the current paper, a possible $\mathbf{L}$ when $\mathbf{M}$ is reordered such that the $\mathbf{M}_{\mathbf{1}}$ is of full rank, thus containing the linearly independent reactions, the $\mathbf{L}$ can be chosen equal to $\mathbf{M}_{1}{ }^{\top}$,

$$
\mathbf{v}=\mathbf{M}_{1}{ }^{\top} \mathbf{C}
$$

\subsection{Simple decomposition through partitioning the stoichiometric matrix}

From section 4.1:

$$
\begin{aligned}
\mathrm{d}(\mathrm{Vc}) / \mathrm{dt} & =\mathbf{N}^{\top} \mathbf{r}(\mathbf{c}, \mathrm{T})+\mathbf{c}_{\text {in }} \mathrm{q}-\mathrm{q} \mathbf{c}(\mathrm{t}) \\
\text { or } \quad \mathrm{d} / \mathrm{dt} \quad & =\mathbf{M}^{\top} \mathbf{r}(\mathbf{c})+1 / \mathrm{T}\left(\mathbf{c}_{\text {in }}-\mathbf{c}\right) ;
\end{aligned}
$$

where $\mathbf{M}^{\mathbf{T}}=\mathbf{N}^{\mathbf{T}} \mathrm{V} ; \mathrm{T}=\mathrm{V} / \mathrm{q}$

Establishing a full rank submatrix $\mathbf{N}_{1}=\mathrm{V} \mathbf{M}_{1}{ }^{\top}$ (section 4.1) of the stoichiometric matrix, then with $\mathbf{M}=\left(\underset{R \times R}{\mathbf{M}_{1}} \mid \underset{\mathrm{R} \times(\mathrm{S}-\mathrm{R})}{\mathbf{M}_{2}}\right)$ the reaction variants can be expressed as follows, by change of variables from the c-vector to the $\mathbf{z}$-vector, given below:

$\mathbf{z}_{1}=\mathbf{c}_{1} \quad\left(\operatorname{dim} \mathbf{c}_{1}=\mathrm{R}\right) ; \mathbf{z}_{10}=\mathbf{c}_{10} ; \operatorname{col} \mathbf{c}=\operatorname{col}\left(\mathbf{c}_{1}, \mathbf{c}_{2}\right) ; \operatorname{dim} \mathbf{c}_{2}=\mathrm{S}-\mathrm{R}$

and the asymptotical invariant is given by 
$\mathbf{z}_{2}=\mathrm{M}_{2}^{\top}\left(\mathrm{M}_{1}^{\top}\right)^{-1} \mathbf{c}_{1} \quad-\mathrm{c}_{2}$, with

$Z_{20}=M_{2}^{\top}\left(M_{1}^{\top}\right)^{-1} c_{10}-c_{20}$

The decomposed set is hence

$\mathrm{d} \mathbf{z}_{1} / \mathrm{dt}=1 / \mathrm{T}\left(\mathbf{z}_{1}-\mathbf{z}_{10}\right)+\mathbf{M}_{1}^{\top} \mathbf{r}\left(\mathbf{z}_{1}, \mathbf{z}_{2}, \mathbf{M}_{1}, \mathbf{M}_{2}\right)$

$\mathrm{dz} \mathrm{z}_{2} / \mathrm{dt}=1 / \mathrm{T}\left(\mathrm{z}_{2}-\mathrm{z}_{20}\right)$

$\mathbf{z}_{\mathbf{2}}$ will be asymptotically invariant, $\mathrm{d} \mathbf{z}_{\mathbf{2}} / \mathrm{dt} \rightarrow \mathbf{0}$ as $\mathrm{t} \rightarrow \infty$

If $\mathbf{z}_{2}(0)$ is located on the submanifold (in a subspace defined by parameters ( $\mathbf{c}_{1}$, $\mathbf{c}_{2}$ ) constrained by $\mathbf{M}_{2}^{\top}\left(\mathbf{M}_{1}^{\top}\right)^{-1} \mathbf{c}_{1}-\mathbf{c}_{2}=0$ ), then $\mathbf{z}_{2}(t)$ will be truly invariant for all $t$ $>0$ when the initial condition $\mathbf{z}_{\mathbf{2}}(\mathbf{0})=\mathbf{0}$. Note that this variable is flow variant if $\mathrm{V} / \mathrm{q}$ is varying with time, hence $\mathbf{z}_{2}$ may generally not be completely invariant.

So it is concluded that for a simple process as a CSTR, decomposition to obtain the asymptotic invariant is straightforward.

\subsection{A step of generalization}

However, the analysis in 4.2 should be extended to check its generality in more general models. In order to make the aforementioned method of analysis of invariance slightly more general when a distributed mass transport phenomenom is present, assume that the reaction kinetics is described as in section 2- 3, and that the decomposition of the stoichiometric matrix, $\mathbf{N}=\left[\begin{array}{lll}\mathbf{N}_{1} & \mathbf{N}_{2}\end{array}\right]$ is such that $\mathbf{M}_{1}{ }^{\top}=$ $\mathrm{N}_{1}{ }^{\mathrm{T}} / \mathrm{V}_{\text {in }}$ has full rank.

Consider mass transport mechanisms represented through a partial spatial differential operator $\boldsymbol{d}$ defined on a spatial domain $\Omega$. For the purpose of simple formal analysis here, we do not consider the boundary conditions (implying that the domain is infinite). The state vector of concentrations $\mathbf{c}$ for a given point in $\Omega$ is embedded in a state space $\mathrm{N}$ of dimension $\mathrm{S}$, so that that system dynamics is described in a $\mathrm{N} \times \Omega$ space, which implies that the state space is infinite-dimensional (a consequence of a distributed system).

$\partial \mathbf{c}_{1} / \partial \mathrm{t}=\delta_{1} \mathbf{c}_{1}+\mathrm{N}_{1}^{\top} \mathrm{r}(\mathbf{c}) ; \operatorname{dim}\left(\mathbf{c}_{1}\right)=\mathrm{R} ;$

$d_{1}$ is defined for operating on $c_{1} \in \Omega$

$\partial \mathbf{c}_{2} / \partial \mathrm{t}=d_{2} \mathbf{c}_{1}+\mathrm{N}_{2}^{\top} \mathrm{r}(\mathbf{c}) ; \operatorname{dim}\left(\mathbf{c}_{2}\right)=S-R ;$

$\delta_{2}$ is defined for operating on $c_{2} \in \Omega$

resulting in

$$
r(\mathbf{c})=\left(N_{1}^{\top}\right)^{-1}\left(\partial \mathbf{c}_{1} / \partial t-d_{1} \mathbf{c}_{1}\right)
$$


$\partial \mathbf{c}_{2} / \partial t-N_{2}^{\top}\left(N_{1}^{\top}\right)^{-1} \partial c_{1} / \partial t=d_{2} c_{2}-N_{2}^{\top}\left(N_{1}^{\top}\right)^{-1} d_{1} c_{1}$

As in the lumped parameter case, now define the "proposed potential asymptotic invariant"

$\mathbf{Z}:=\mathbf{c}_{2}-\mathrm{N}_{2}^{\top}\left(\mathrm{N}_{1}^{\top}\right)^{-1} \mathbf{C}_{1}$

which gives

$\partial \mathbf{z} / \partial \mathrm{t}=\delta_{2} \mathbf{c}_{2}-\mathrm{N}_{2}^{\top}\left(\mathrm{N}_{1}^{\top}\right)^{-1} d_{1} \mathbf{c}_{1}$

The reaction invariant property is apparently destroyed by the partial differential operators $\delta_{2}$ and $d_{1}$ in eq(28).

The breakdown of the invariance property caused by distributed flow (such as diffusion) may perhaps be even more clearly understood and demonstrated mathematically if the operators $d_{i}$ are discretized, assuming diffusion on an infinite line with distance parameter $\ell$, just for simplification and demonstration. The finite elements are of length $\Delta h$. For simplicity, assume $\Delta h$ is of unit length. Then the typical element when the diffusion term $\partial^{2}(\mathbf{c}) / \partial \ell^{2}$ has the simplest discrete representation, at some discrete point $\mathrm{k}$ along $\ell$,

$\partial^{2}\left(\mathbf{c}_{\mathrm{k}}\right) / \partial \ell^{2} \approx \mathbf{c}_{\mathrm{k}-1}-2 \mathbf{c}_{\mathrm{k}}+\mathbf{c}_{\mathrm{k}}$

and

$\mathbf{c}_{\mathrm{j}}=0$ for $\mathrm{j}<\mathrm{k}-1$ and $\mathrm{j}>\mathrm{k}+1$

where (31)-(32) represent elements in a band diagonal matrix. It is observed that this represents the forward and backward transport of the diffusing $\mathbf{c}_{\mathrm{k}}$ elements, assuming for convenience that the diffusion coefficient is unity. Hence, the simplest discrete representation with diffusion in one spatial direction and chemical reaction, is (for the $R$ reaction equations)

$\mathrm{d}\left(\mathbf{c}_{1}, \mathrm{k}\right) / \mathrm{dt}=\mathbf{c}_{1, \mathrm{k}-1}-2 \mathbf{c}_{1, \mathrm{k}}+\mathbf{c}_{1, \mathrm{k}+1}+\mathbf{N}_{1}^{\top} \mathbf{r}\left(\mathbf{c}_{\mathrm{k}}\right)$

$\mathbf{r}$ is defined as a rate, i.e. of component material, weighted by the stoichiometric coefficients, created per unit volume and time, to result in the converted material components in the elements of vector $\mathbf{c}_{1}$. For $\mathbf{c}_{2}$, the S-R remaining equations,

$\mathrm{d}\left(\mathbf{c}_{2}, \mathrm{k}\right) / \mathrm{dt}=\mathbf{c}_{2, \mathrm{k}-1}-2 \mathbf{c}_{2, \mathrm{k}}+\mathbf{c}_{2, \mathrm{k}+1}+\mathbf{N}_{\mathbf{2}}^{\top} \mathbf{r}\left(\mathbf{c}_{\mathrm{k}}\right)$

Then

$\mathrm{d} / \mathrm{dt}\left(\mathbf{c}_{\mathbf{2}, \mathbf{k}}-\mathbf{N}_{\mathbf{2}}^{\top}\left(\mathbf{N}_{\mathbf{1}}^{\top}\right)^{-1} \mathbf{c}_{\mathbf{1}, \mathbf{k}}\right)=\Delta^{2} \mathbf{c}_{\mathbf{2}}(\mathrm{k})-\mathbf{N}_{\mathbf{2}}^{\top}\left(\mathbf{N}_{\mathbf{1}}^{\top}\right)^{-1} \Delta^{2} \mathbf{c}_{\mathbf{1}}(\mathrm{k})$

where 
$\Delta^{2} \mathbf{c}_{i}(\mathrm{k})=\mathbf{c}_{\mathrm{i}, \mathrm{k}-1}-2 \mathbf{c}_{\mathrm{i}, \mathrm{k}}+\mathbf{c}_{\mathrm{i}, \mathrm{k}+1} \quad \mathrm{i}=1,2$

The "proposed potential invariant" $\mathbf{z}_{\mathbf{k}}=\mathbf{c}_{2, \mathbf{k}}-\mathrm{N}_{2}^{\top}\left(\mathrm{N}_{1}^{\top}\right)^{-1} \mathbf{c}_{1, \mathbf{k}}$ is described by a set of d.e.where the r.h.s. of eq(35) can't generally be reduced to an expression containing $\mathbf{z}_{\mathbf{k}}$ only.

\section{An alterative approximation in modelling}

A promising road to be able to obtain asymptotic invariants in modeling in a distributed system is: Consider continuous stirred tank reactors coupled in series,

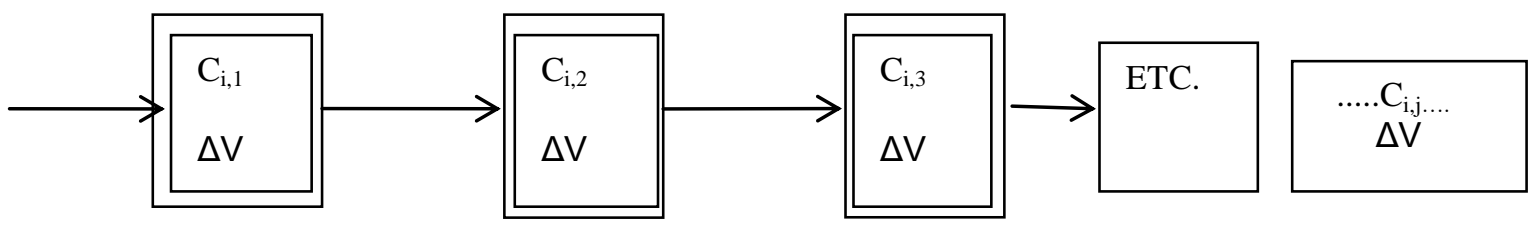

$\mathrm{T}=\Delta \mathrm{V} / \mathrm{q} \quad \mathrm{k}=1 \quad \mathrm{k}=2 \quad \mathrm{k}=3 \ldots \ldots \ldots$

It is known from standard literature on reactor theory, that in the case of dispersed nonreacting matter flow in a tubular reactor, with axial diffusion, the input/output impulse response may be approximated by a series of CSTRs (H.Scott Fogler 2004; Md Firoz Kalam 2016). The approximation will be good when the number of CSTRs is greater than some figure depending on the accuracy of the fit.

In this case, for each CSTR element,

$\mathrm{d} / \mathrm{dt}\left(\mathbf{c}_{\mathrm{i}, \mathrm{k}}\right)=1 / \Delta \mathrm{T}\left(\mathbf{c}_{\mathrm{i}, \mathrm{k}-1}-\mathbf{c}_{\mathrm{i}, \mathrm{k}}\right)+\mathrm{N}_{\mathrm{i}}^{\top} \mathbf{r}\left(\mathbf{c}_{\mathrm{k}}\right) ; \mathrm{i}=1,2 \quad ; \mathrm{k}=1,2,3,4 \ldots$

By solving for $r$ in the first equation for $i=1$, when $N_{1}$ is of full rank, then composing the expression for the asymptotic invariant,

$\mathrm{d} / \mathrm{dt}\left(\mathbf{c}_{2, \mathbf{k}}-\mathrm{N}_{2}^{\top}\left(\mathrm{N}_{1}^{\top}\right)^{-1} \mathbf{c}_{1, \mathrm{k}}\right)=1 / \Delta \mathrm{T}\left(\mathbf{c}_{2}(\mathrm{k})-\mathrm{N}_{2}^{\top}\left(\mathrm{N}_{1}^{\top}\right)^{-1} \mathbf{c}_{1}(\mathrm{k})\right)$

Or, with

$\mathbf{Z}_{\mathbf{k}}=\mathbf{c}_{2}(\mathrm{k})-\mathrm{N}_{2}^{\top}\left(\mathrm{N}_{1}^{\top}\right)^{-1} \mathbf{c}_{1}(\mathrm{k})$

then

$\mathrm{d} / \mathrm{dt} \mathbf{z}_{\mathrm{k}}=-1 / \Delta \mathrm{T} \mathbf{z}_{\mathrm{k}}+1 / \Delta \mathrm{T} \mathbf{z}_{\mathrm{k}-1 ;} ; \quad \Delta \mathrm{T}=\Delta \mathrm{V} / \mathrm{q}$

Cleary, at each stage $k$, there is no true invariant, since the state is input flow variant , through $(\mathrm{q} / \Delta \mathrm{V}) \mathbf{z}_{\mathrm{k}-1}$ if $\mathrm{q}=\mathrm{q}(\mathrm{t})$. However, since each $\mathbf{z}_{\mathrm{k}}$ is flow variant, it means if $\mathbf{q}$ is constant, each $\mathbf{z}_{\mathbf{k}}$ will asymptotically approach $\mathbf{z}_{\mathbf{k}}=\mathbf{z}_{\mathbf{0}}=$ constant if $\mathbf{z}_{0}$ 
on the reactor chain input side is a constant. So all $\mathbf{z}_{\mathrm{k}}, \mathrm{k}=1,2,3, \ldots$ will be asymptotically flow and reaction invariant under these conditions.

In this way, the potential nonexistence of invariance has been circumvented by applying a different discretized model of a tubular reactor with axial diffusion, a model which may be a good input/output approximation to the distributed model. The tanks-in-series model is simple and has computational advantages, although its physical basis is not as clear as the tubular diffusion model.

Thus the analysis show that invariants contained in process models can - through a discrete approximation - indeed be extended to cover processes with diffusion. Thus the strong statement of Asbjørnsen (1972) on invariants to simplify and extend the application of chemical process models is more general than perhaps originally thought.

A weak point is that the discrete approximation above has not been generally proved to be robust or accurate enough with reactions included, even though the nonreacting approximation is good; this is documented in textbooks of chemical engineering. However, there are also many studies published with reactions, that show that the CSTR approximation with various chemical reactions present, is applicable and useful. CSTRs cannot model high axial dispersion well, though. See for instance Sayer, C., Giudici, R. (2002), and I.M. Abu-Reesh (2003); the latter has compared tanks-in-series with axial dispersion for enzyme reactions. However, the two modelling approaches compare well for high Peclet numbers (i.e. high advective transport rate compared to diffusive transport rate).

\section{CONCLUSIONS}

Dynamic model reduction techniques based on the decomposition of the stoichiometric matrix to find the chemical invariant, break down if axial diffusion is present in a tubular reactor.

Straightforward discretization of the partial differential operator does indeed show that the resulting discrete dynamic model cannot generally be partioned to obtain the reaction variant vector and the reaction invariant (asymptotic) vector. However, the paper demonstrate that, if the diffusional tubular reactor is discretely and approximatively represented by tanks-in-series, then matrix approaches to successfully find the chemical variant and invariant vectors of the resulting chemical process model is possible.

\section{Literature}

Cayley, A.:On the theory of linear transformations. Cambridge math. J. Vol 4,1845, pp193-209

Gorban A.N., I.V.Karlin, Springer (2005):Invariant Manifolds for Physical and Chemical Kinetics. 
Chevalier et.al (1992):Astrophysical Journal, 392, 118

Aris, R. (1965), Introduction to the analysis of chemical reactors, Englewood Cliffs, NJ.

Aris, R. (1968): "Prolegomena to the Rational Analysis of Systems of Chemical Reactions", Archive for Rational Mechanics and Analysis 27 (5), 356-364), January 1968.

Amundson, N.R., (1966) Mathematical methods in Chemical Engineering I, New York,Prentice Hall

Froment G.F. (1967), Industrial and Engineering Chemistry, 59(2), 18-27.

Fjeld M. (1968), Chem.Eng.Sci. 23, pp 565-573 ( submitted to the journal in 1967). $\boldsymbol{\xi}(\mathrm{t})=\left(\mathbf{M}^{\mathrm{T}}\right)^{-1}\left(\mathbf{c}(\mathrm{t})-\mathbf{C}_{\mathbf{0}}\right)$

Fjeld M. (1969), Chem.Eng.Sci.24, pp1023-1024

Asbjørnsen O.A \& M. Fjeld (1970), 25, pp 1627-1636

Fjeld M (1971), Stability and Control of Periodic Processes, Doctor Technicae

Thesis, Norwegian Inst.of technology, 71-52-W.

Fjeld M. et al.(1974), Chem.Eng. Sci. , vol 29, pp.1917-1926.

Aggarwal et al (2007), AIChE Annual Meeting, No.7, 2007

Waller, K., P.M.Makila,(1981), Ind.\&Chem.Proc.Des.and Developm. 20,1-11

Asbjørnsen, O.A. (1972): Chem.Eng.Sci. , vol 27,pp 709-717

Asbjørnsen, O.A. (1997) : A I ChE Journal Vol 43, no.4, April 1997,pp 1082-1090

I.M. Abu-Reesh et al (2003) Ind.\& Chem. Res. 42,Issue 22, 5495-5505

Fogler, H. Scott, Elements of Chemical Reaction Engineering. Chem.Engng.

University of Michigan, Ann Arbor. Prentice-Hall of India, $3^{\text {rd }}$ ed. 2004.

Kalam, Md Firoz, Modelling and Simulation of Real Reactor, July 2016, Hochschule Bremerhaven.

Sayer, C., Giudici, R (2002),Braz.J.of Chem.Eng., vol 19, no1. 